\title{
Structure preserving iterative methods for periodic projected Lyapunov equations and their application in model reduction of periodic descriptor systems
}

\author{
Peter Benner · Mohammad-Sahadet \\ Hossain
}

\begin{abstract}
In this paper, we develop structure preserving iterative schemes to solve the periodic discrete-time projected Lyapunov equations associated to analysis and design of discrete-time descriptor systems exploiting the reflexive generalized inverses of the periodic matrices associated with these systems. In particular, we extend the Smith method to solve the large scale projected periodic discrete-time algebraic Lyapunov equations in lifted form. A low-rank version of this method is also presented, avoiding the explicit lifted formulation and working directly with the periodic matrix coefficients. Moreover, we consider an application of the Lyapunov solvers in balanced truncation model reduction of periodic discrete-time descriptor systems. Numerical results are given to illustrate the efficiency and accuracy of the proposed methods.
\end{abstract}

Keywords Periodic descriptor systems - Lifted state space representation . Periodic projected Lyapunov equations . Smith iteration - Model order reduction

\section{Introduction}

Periodic systems and control theory have received a lot of attention in the last few decades because they have wide applications in many areas of science and engineering, specially in the areas where the periodic control is deserved, such as aerospace realm, control of industrial processes and communication systems, modeling of periodic time-varying filters and networks $[13,17,16,29]$.

P. Benner

Max Planck Institute for Dynamics of Complex Technical Systems, Sandtorstr. 1, D-39106 Magdeburg, Germany

E-mail: benner@mpi-magdeburg.mpg.de

M.-S. Hossain

Department of Mathematics and Physics, North South University, Dhaka, Bangladesh

E-mail: mohammad.hossain@northsouth.edu 
In this paper, we consider linear time-varying (LTV) discrete-time descriptor systems of order $\overline{\mathbf{n}}=\left(n_{0}, n_{1}, \ldots, n_{K-1}\right)$, as

$$
E_{k} x_{k+1}=A_{k} x_{k}+B_{k} u_{k}, \quad y_{k}=C_{k} x_{k}, \quad k \in \mathbb{Z},
$$

where $E_{k} \in \mathrm{R}^{\mu_{k} \times n_{k+1}}, A_{k} \in \mathrm{R}^{\mu_{k} \times n_{k}}, B_{k} \in \mathrm{R}^{\mu_{k} \times m_{k}}, C_{k} \in \mathrm{R}^{p_{k} \times n_{k}}$ are the system matrices, $x_{k} \in \mathrm{R}^{n_{k}}$ is the (generalized) state or descriptor vector, $u_{k} \in \mathrm{R}^{m_{k}}$ is the control input, and $y_{k} \in \mathrm{R}^{p_{k}}$ is the output. The system matrices are periodic with a period $K \geq 1$, and $\sum_{k=0}^{K-1} \mu_{k}=\sum_{k=0}^{K-1} n_{k}=\mathbf{n}$, $\sum_{k=0}^{K-1} m_{k}=\mathbf{m}$ as well as $\sum_{k=0}^{K-1} p_{k}=\mathbf{p}$. If all $E_{k}$ are nonsingular, then (1) can be transformed into a periodic standard system.

Efficient numerical methods for computing poles and zeros, $\mathbb{L}_{\infty}$-norm, minimal and balanced realizations have been developed for such systems [6,33-35]. Model reduction of such systems using balanced truncation has been proposed in [1]. All these methods are restricted to problems of small or medium size $[1,30]$. Much attention has recently been devoted to the iterative solution of large-scale sparse Lyapunov equations. Iterative solvers for projected generalized Lyapunov equations have been proposed in[24,25]. On the other hand, an extension of the Smith method and the block-Arnoldi based Krylov subspace method to standard periodic Lyapunov equations has been presented in [11]. But,these methods cannot be directly applied to the projected periodic Lyapunov equations [2].

Analysis and reduced order modeling of periodic discrete-time descriptor systems may require to invert the system matrices in some appropriate sense. A special form of the generalized inverses, known as (1,2)-inverses, of periodic systems in the descriptor form has been considered in [31], which works on the corresponding lifted form of the associated system pencil of the periodic system.

In this paper, we discuss the computation of the generalized inverses of periodic discrete-time descriptor systems using the left and right deflating projectors associated with the eigenstructures of the periodic matrix pairs. This technique has been implemented in [25] for continuous-time descriptor system to compute the solution of the corresponding projected Lyapunov equations. We will generalize the idea of [25] for the discrete periodic setting and use those periodic inverses to compute the solutions of the periodic projected Lyapunov equations. Moreover, we reformulate the Smith method to solve the large projected periodic discrete-time algebraic Lyapunov equations in lifted form. The block diagonal structure of the periodic solutions is preserved in every Smith iteration step which is one of the challenging task in many of the iterative computations in the periodic setting. It should be noted that generalized versions of the ADI method and the Smith method have been proposed in [2] for the solution of projected periodic Lyapunov equations. But, the methods fail to preserve the block diagonal structure during the iteration, and it is only achieved upon convergence.

The rest of the paper is organized as follows. In Section 2, we briefly review discrete-time periodic descriptor systems and their cyclic lifted representations. We also study the causal and noncausal decomposition of the periodic 
descriptor systems. In Section 3, we introduce the periodic matrix equations under consideration and review the different techniques for the solutions of those matrix equations associated to the causal and noncausal systems. We also discuss the challenges of iterative techniques used in computing the structure preserving solution of those matrix equations. In Sections 4, we discuss the generalized inverses of periodic discrete-time descriptor systems using the left and right deflating projectors associated with the eigenstructures of the periodic matrix pairs. Iteratively solving of the causal and noncausal lifted Lyapunov equations exploiting the generalized inverses is discussed in Sections 5 and 6, respectively. Low-rank versions of these methods are also presented, which avoid the explicit lifted formulation and work directly with the period matrix coefficients. A balanced truncation model reduction method for periodic descriptor systems is considered in Section 7. Section 8 contains numerical examples that illustrate the properties of the described iterative methods for projected Lyapunov equations and their application to model reduction. Some conclusions are given in Section 9.

\section{Periodic descriptor systems}

Lifted representations $[4,7,33]$ of periodic systems are the analogous timeinvariant representations of the periodic systems. Using the lifting isomorphism one can exploit the theory of time-invariant systems for the analysis and control of periodic systems, provided that the results achieved can be easily re-interpreted in a periodic framework.

\subsection{Cyclic lifted representation of periodic systems}

We consider here the cyclic lifted representation which was introduced first for standard periodic systems in [17]. The essence of the cyclic lifted system is putting inputs, states and outputs of the original LTV descriptor system at cyclic places of those of the lifted LTI system.

The cyclic lifted representation of the periodic descriptor system (1) is given by

$$
\mathcal{E} \mathcal{X}_{k+1}=\mathcal{A} \mathcal{X}_{k}+\mathcal{B} \mathcal{U}_{k}, \quad \mathcal{Y}_{k}=\mathcal{C} \mathcal{X}_{k}
$$

where

$$
\begin{gathered}
\mathcal{E}=\operatorname{diag}\left(E_{0}, E_{1}, \ldots, E_{K-1}\right), \quad \mathcal{B}=\operatorname{diag}\left(B_{0}, B_{1}, \ldots, B_{K-1}\right), \\
\mathcal{A}=\left[\begin{array}{cccc}
0 & \ldots & 0 & A_{0} \\
A_{1} & & & 0 \\
& \ddots & & \vdots \\
0 & & A_{K-1} & 0
\end{array}\right], \quad \mathcal{C}=\left[\begin{array}{cccc}
0 & \ldots & 0 & C_{0} \\
C_{1} & & & 0 \\
& \ddots & & \vdots \\
0 & & C_{K-1} & 0
\end{array}\right] .
\end{gathered}
$$


The descriptor vector, system input and output of (2) are related to those of (1) via

$$
\mathcal{X}_{k}=\left[\begin{array}{c}
x_{1} \\
\vdots \\
x_{K-1} \\
x_{0}
\end{array}\right], \quad \mathcal{U}_{k}=\left[\begin{array}{c}
u_{0} \\
u_{1} \\
\vdots \\
u_{K-1}
\end{array}\right], \quad \mathcal{Y}_{k}=\left[\begin{array}{c}
y_{0} \\
y_{1} \\
\vdots \\
y_{K-1}
\end{array}\right]
$$

respectively. The transfer function of the lifted system (2) can be rewritten as

$$
\mathcal{H}(z)=\mathcal{C}(z \mathcal{E}-\mathcal{A})^{-1} \mathcal{B} .
$$

The cyclic lifted system (2) describes the eigenstructure and system dynamics of the LTV discrete-time descriptor system (1). Regularity of the periodic matrix pairs $\left\{\left(E_{k}, A_{k}\right)\right\}_{k=0}^{K-1}$ implies the regularity of the cyclic matrix pair $(\mathcal{E}, \mathcal{A})[14,22,13]$. The reverse argument also holds true.

The cyclic matrix pencil $z \mathcal{E}-\mathcal{A}$ is said to be regular when $\operatorname{det}(z \mathcal{E}-\mathcal{A}) \neq 0$ for some $z \in \mathbb{C}$. The cyclic lifted system (2) is asymptotically stable iff $z \mathcal{E}-\mathcal{A}$ is regular and all its finite eigenvalues lie inside the unit circle. System (1) is asymptotically stable if and only if the corresponding cyclic lifted system (2) is asymptotically stable.

\subsection{Spectral projectors for periodic matrix pairs}

In the descriptor setting, the $E_{k}$ in (1) are singular for $k=0,1, \ldots, K-1$, and analysis and modeling of system (1) can be proceed by separating the causal and the noncausal parts of the periodic descriptor system (1). The periodic Kronecker canonical form $[27,23,6]$ of the matrix pairs $\left\{\left(E_{k}, A_{k}\right)\right\}_{k=0}^{K-1}$ for $k=0,1, \ldots, K-1$, can be used for this separation.

$$
U_{k} E_{k} V_{k+1}=\left[\begin{array}{cc}
I_{n_{k+1}^{f}} & 0 \\
0 & E_{k}^{b}
\end{array}\right], U_{k} A_{k} V_{k}=\left[\begin{array}{cc}
A_{k}^{f} & 0 \\
0 & I_{n_{k}^{\infty}}
\end{array}\right],
$$

where $U_{k}, V_{k}$ are nonsingular. Then the left and right spectral projectors $P_{l}(k)$ and $P_{r}(k)$, for $k=0,1, \ldots, K-1$, can be represented as [2,6,24],

$$
P_{l}(k)=U_{k}^{-1}\left[\begin{array}{cc}
I_{n_{k+1}^{f}} & 0 \\
0 & 0
\end{array}\right] U_{k}, \quad P_{r}(k)=V_{k}\left[\begin{array}{cc}
I_{n_{k}^{f}} & 0 \\
0 & 0
\end{array}\right] V_{k}^{-1},
$$

respectively. The index $\rho$ of the periodic descriptor system (1) and the index $\nu$ of the lifted system (2) are related via $\nu \leq K \rho[2,6,24]$. 


\section{Periodic matrix equations and their solutions}

Stability analysis and model reduction of periodic systems are strongly related to the matrix equations associated with the systems. For disctere-time periodic descriptor systems, they are well known as generalized projected periodic discrete-time algebraic Lyapunov equations (PPDALEs). It has been shown in $[2,6]$ that the periodic Gramians of the asymptotically stable discrete-time descriptor systems (1) satisfy the PPDALEs with special right-hand sides. The causal and noncausal reachability Gramians $X_{k}$ and $\hat{X}_{k}$ are the unique symmetric, positive semidefinite periodic solutions of the PPDALEs

$$
\begin{aligned}
A_{k} X_{k} A_{k}^{T}-E_{k} X_{k+1} E_{k}^{T} & =-P_{l}(k) B_{k} B_{k}^{T} P_{l}(k)^{T}, \\
X_{k} & =P_{r}(k) X_{k} P_{r}(k)^{T},
\end{aligned}
$$

and

$$
\begin{aligned}
A_{k} \hat{X}_{k} A_{k}^{T}-E_{k} \hat{X}_{k+1} E_{k}^{T} & =Q_{l}(k) B_{k} B_{k}^{T} Q_{l}(k)^{T}, \\
\hat{X}_{k} & =Q_{r}(k) \hat{X}_{k} Q_{r}(k)^{T},
\end{aligned}
$$

respectively, where $X_{K}=X_{0}, \hat{X}_{K}=\hat{X}_{0}$. Note that $P_{l}(k), P_{r}(k)$, for $k=$ $0,1, \ldots, K-1$, are the spectral projectors onto the $k$-th left and right deflating subspaces of the periodic matrix pairs $\left\{\left(E_{k}, A_{k}\right)\right\}_{k=0}^{K-1}$ corresponding to the finite eigenvalues $[1,6]$, and $Q_{l}(k)=I-P_{l}(k)$ and $Q_{r}(k)=I-P_{r}(k)$ in $(8)$.

Similarly, the causal and noncausal observability Gramians $Y_{k}$ and $\hat{Y}_{k}$ are the unique symmetric, positive semidefinite periodic solutions of the PPDALEs

$$
\begin{aligned}
A_{k}^{T} Y_{k+1} A_{k}-E_{k-1}^{T} Y_{k} E_{k-1} & =-P_{r}^{T}(k) C_{k}^{T} C_{k} P_{r}(k) \\
Y_{k} & =P_{l}(k-1)^{T} Y_{k} P_{l}(k-1)
\end{aligned}
$$

and

$$
\begin{aligned}
A_{k}^{T} \hat{Y}_{k+1} A_{k}-E_{k-1}^{T} \hat{Y}_{k} E_{k-1} & =Q_{r}(k)^{T} C_{k}^{T} C_{k} Q_{r}(k), \\
\hat{Y}_{k} & =Q_{l}(k-1)^{T} \hat{Y}_{k} Q_{l}(k-1),
\end{aligned}
$$

respectively, where $Y_{K}=Y_{0}, \hat{Y}_{K}=\hat{Y}_{0}$.

The numerical solution of (7) has been considered in [6] for time-varying matrix coefficients. The method proposed there is based on an initial reduction of the periodic matrix pairs $\left\{\left(E_{k}, A_{k}\right)\right\}_{k=0}^{K-1}$ to the generalized periodic Schur form $[12,32]$ and on solving the resulting generalized periodic Sylvester and Lyapunov equations. As a result, the method is computationally expensive and not suitable for large scale problems.

An efficient approach which works with the cyclic lifted representation of (1) and the corresponding lifted form of (7) has been considered in [1]. Following the work of [1], the PPDALEs (7) and (8) are equivalent to the following projected lifted discrete-time algebraic Lyapunov equations (PLDALEs)

$$
\begin{aligned}
\mathcal{A} \mathcal{X} \mathcal{A}^{T}-\mathcal{E} \mathcal{X} \mathcal{E}^{T}=-\mathcal{P}_{l} \mathcal{B B} \mathcal{B}^{T} \mathcal{P}_{l}^{T}, & \mathcal{X}=\mathcal{P}_{r} \mathcal{X} \mathcal{P}_{r}^{T}, \\
\mathcal{A} \hat{\mathcal{X}} \mathcal{A}^{T}-\mathcal{E} \hat{\mathcal{X}} \mathcal{E}^{T}=\mathcal{Q}_{l} \mathcal{B B} \mathcal{B}^{T} \mathcal{Q}_{l}^{T}, & \hat{\mathcal{X}}=\mathcal{Q}_{r} \hat{\mathcal{X}} \mathcal{Q}_{r}^{T},
\end{aligned}
$$


respectively, where $\mathcal{E}, \mathcal{A}$ and $\mathcal{B}$ are as in (3), and

$$
\mathcal{X}=\operatorname{diag}\left(X_{1}, \ldots, X_{K-1}, X_{0}\right), \quad \hat{\mathcal{X}}=\operatorname{diag}\left(\hat{X}_{1}, \ldots, \hat{X}_{K-1}, \hat{X}_{0}\right) .
$$

In that case, the projectors in lifted forms are given by

$$
\begin{array}{ll}
\mathcal{P}_{l}=\operatorname{diag}\left(P_{l}(0), P_{l}(1), \ldots, P_{l}(K-1)\right), & \mathcal{Q}_{l}=I-\mathcal{P}_{l}, \\
\mathcal{P}_{r}=\operatorname{diag}\left(P_{r}(1), \ldots, P_{r}(K-1), P_{r}(0)\right), & \mathcal{Q}_{r}=I-\mathcal{P}_{r} .
\end{array}
$$

The matrices $\mathcal{X}$ and $\hat{\mathcal{X}}$ are called the causal and noncausal reachability Gramians of the lifted system (2). A similar result can also be stated for the causal and noncausal observability Gramians [1].

In practice, one should avoid these direct methods for large-scale problems because the computational complexity for solving a Lyapunov equation of the form (11) or (12) using direct methods is at least of order $\left(\mathcal{O}\left(K n_{\max }^{3}\right)\right)$, where $n_{\max }=\max \left(n_{k}\right)$, and they require extensive storage. Therefore, iterative methods have been developed for the solutions of such equations.

\subsection{Revised iterative methods for solutions of PLDALEs}

Iteratively solving (7) and (8) using their corresponding lifted structures, i.e., (11) and (12), has been considered in [2]. A generalized version of the alternating direction implicit (ADI) method and the Smith method is proposed there for the solutions of (11) and (12), respectively. Note that, for the descriptor system (1), the matrix $\mathcal{E}=\operatorname{diag}\left(E_{0}, \ldots, E_{K-1}\right)$ is singular. However, both the ADI and Smith iterations fail to converge for the resulting Lyapunov equations since the iteration operator of the ADI and the Smith iterations, in this case, does not have spectral radius less than one. This problem has been circumvented by considering a generalized Cayley transformation given by

$$
\mathfrak{C}(\mathcal{E}, \mathcal{A})=\lambda(\mathcal{A}-\mathcal{E})-(\mathcal{A}+\mathcal{E})
$$

see, e.g., [15]. This transformation transfers the PLDALE (11) to an equivalent projected continuous-time algebraic Lyapunov equation (PCALE)

$$
\mathbf{E} \mathcal{X} \mathbf{A}^{T}+\mathbf{A} \mathcal{X} \mathbf{E}^{T}=-2 \mathcal{P}_{l} \mathcal{B} \mathcal{B}^{T} \mathcal{P}_{l}^{T}, \quad \mathcal{X}=\mathcal{P}_{r} \mathcal{X} \mathcal{P}_{r}^{T},
$$

where $\lambda \mathbf{E}-\mathbf{A}=\lambda(\mathcal{A}-\mathcal{E})-(\mathcal{A}+\mathcal{E})$ is the Cayley-transformed pencil. The finite eigenvalues of $\lambda \mathcal{E}-\mathcal{A}$ lying inside the unit circle are mapped to the finite eigenvalues of $\lambda \mathbf{E}-\mathbf{A}$ in the open left half-plane, and the eigenvalue of $\lambda \mathcal{E}-\mathcal{A}$ at infinity is mapped to $\lambda=1$.

The solutions of the PLDALE (11) and the PCALE (14) are identical and have the block diagonal structure. Therefore, it is desired that the iterative solution of (14) will have the block diagonal structure analogous to (11) at each ADI iteration step. Unfortunately, the case is not so. It is observed that the generalized ADI method does not preserve the block diagonal structure at every ADI iteration step due to the specific structure of the matrices $\mathbf{E}$ 
and $\mathbf{A}$, although the approximate Gramian is block diagonal [2]. Therefore, we use the concept of reflexive generalized inverses of periodic matrix pairs associated with the periodic system to preserve the block diagonal structure of the approximate solution at each iteration step.

\section{Reflexive generalized inverses of periodic matrices}

For periodic systems, the inversion problem has been introduced in $[10,19]$ by exploiting the concept of left and right invertibility of system (1) in the standard case (i.e., with $E_{k}=I_{n_{k+1}}$ ). A generalization of that concept, which computes the generalized inverses of periodic descriptor systems via the corresponding lifted representation has been considered in [32]. A special class of generalized inverse, called reflexive generalized inverse, of the system pencil has been proposed in [25] to find the solutions of projected continuous-time algebraic Lyapunov equations using Krylov subspace methods, and also in [3] for computing a partial realization for descriptor systems. Similar inverses have been proposed in [31] to compute generalized inverses of rational matrices in descriptor state-space representation, and named there (1,2)-inverse as they satisfy the first two Moore-Penrose conditions. Details of these definitions can be found in [5]. Analogous to [25], we can find the reflexive generalized inverses for $E_{k}$ with respect to the projectors $P_{l}(k)$ and $P_{r}(k+1)$ as

$$
\bar{E}_{k}=V_{k+1}\left[\begin{array}{cc}
I_{n_{k+1}^{f}} & 0 \\
0 & 0
\end{array}\right] U_{k}
$$

for $k=0,1, \ldots, K-1$. Moreover, the reflexive generalized inverses satisfy the relations

$$
\bar{E}_{k} E_{k} \bar{E}_{k}=\bar{E}_{k}, \quad E_{k} \bar{E}_{k}=P_{l}(k), \quad \bar{E}_{k} E_{k}=P_{r}(k+1),
$$

for $k=0,1, \ldots, K-1$. These reflexive generalized inverses of $E_{k}$ for $k=$ $0,1, \ldots, K-1$ will be exploited in the next two sections to find the block diagonal approximate solutions of (11) and (12) using the Smith iterative method.

\section{Generalized Smith method for causal PLDALEs}

Consider again the PLDALE (11). Multiplying it from the left and right by $\overline{\mathcal{E}}$, and $(\overline{\mathcal{E}})^{T}$, we get

$$
\mathcal{P}_{r} \mathcal{X} \mathcal{P}_{r}^{T}-\overline{\mathcal{E}} \mathcal{A} \mathcal{X} \mathcal{A}^{T}(\overline{\mathcal{E}})^{T}=\overline{\mathcal{E}} \mathcal{P}_{l} \mathcal{B} \mathcal{B}^{T} \mathcal{P}_{l}^{T}(\overline{\mathcal{E}})^{T}, \mathcal{X}=\mathcal{P}_{r} \mathcal{X} \mathcal{P}_{r}^{T}
$$

where $\overline{\mathcal{E}} \mathcal{E}=\mathcal{P}_{r}$ by the definition of the reflexive generalized inverse, and $\overline{\mathcal{E}}=$ $\operatorname{diag}\left(\bar{E}_{0}, \bar{E}_{1}, \cdots, \bar{E}_{K-1}\right)$. Equation (17) can be written in a more usual form as

$$
\mathcal{X}-(\overline{\mathcal{E}} \mathcal{A}) \mathcal{X}(\overline{\mathcal{E}} \mathcal{A})^{T}=\mathcal{P}_{r} \overline{\mathcal{E}} \mathcal{B}\left(\mathcal{P}_{r} \overline{\mathcal{E}} \mathcal{B}\right)^{T}, \mathcal{X}=\mathcal{P}_{r} \mathcal{X} \mathcal{P}_{r}^{T}
$$


where $\mathcal{X}=\mathcal{P}_{r} \mathcal{X} \mathcal{P}_{r}^{T}$. Note that in the above representation, we use the relation $\mathcal{P}_{r} \overline{\mathcal{E}}=\overline{\mathcal{E}} \mathcal{P}_{l}$. Clearly, $\mathcal{P}_{l}$ and $\mathcal{P}_{r}$ are the spectral projectors onto the invariant subspace of the matrix $\overline{\mathcal{E}} \mathcal{A}$ corresponding to eigenvalues inside the unit circle. Such an equation can be solved by the Smith method $[18,24]$ given by

$$
\begin{aligned}
& \mathcal{X}_{1}=\mathcal{P}_{r} \overline{\mathcal{E}} \mathcal{B}\left(\mathcal{P}_{r} \overline{\mathcal{E}} \mathcal{B}\right)^{T} \\
& \mathcal{X}_{\ell}=\mathcal{P}_{r} \overline{\mathcal{E}} \mathcal{B}\left(\mathcal{P}_{r} \overline{\mathcal{E}} \mathcal{B}\right)^{T}+(\overline{\mathcal{E}} \mathcal{A}) \mathcal{X}_{\ell-1}(\overline{\mathcal{E}} \mathcal{A})^{T}
\end{aligned}
$$

Then the unique solution $\mathcal{X}$ of (18) can be approximated using the Smith iterations

$$
\mathcal{X}_{i}=\sum_{\ell=0}^{i-1}(\overline{\mathcal{E}} \mathcal{A})^{\ell} \mathcal{P}_{r} \overline{\mathcal{E}} \mathcal{B} \mathcal{B}^{T} \overline{\mathcal{E}}^{T} \mathcal{P}_{r}^{T}\left((\overline{\mathcal{E}} \mathcal{A})^{T}\right)^{\ell} .
$$

Therefore, the Cholesky factor $\mathcal{R}_{i}$, where $\mathcal{X}_{i}=\mathcal{R}_{i} \mathcal{R}_{i}^{T}$, is given by

$$
\mathcal{R}_{i}=\left[\mathcal{P}_{r} \overline{\mathcal{E}} \mathcal{B},(\overline{\mathcal{E}} \mathcal{A}) \mathcal{P}_{r} \overline{\mathcal{E}} \mathcal{B}, \ldots,(\overline{\mathcal{E}} \mathcal{A})^{i-1} \mathcal{P}_{r} \overline{\mathcal{E}} \mathcal{B}\right]
$$

Remark 1 At each iteration step $i$ in (21), we do not have an analogous block diagonal structure as given in (11). Note that $\mathcal{X}=\operatorname{diag}\left(X_{1}, \ldots, X_{K-1}, X_{0}\right)$, and $X_{k}=R_{k} R_{k}^{T}$ for $k=0,1, \ldots, K-1$. Hence, we demand to compute the block diagonal Cholesky factor $\mathcal{R}_{i}=\operatorname{diag}\left(R_{1, i}^{b}, \ldots, R_{K-1, i}^{b}, R_{0, i}^{b}\right)$ at each iteration step $i$ of (21), where $R_{k, i}^{b}$ collocates all the iterative counterparts of the computed $R_{k}$ at the $i$-th iteration steps for $k=0,1, \ldots, K-1$, and $X_{k}=R_{k} R_{k}^{T} \approx R_{k, i}^{b}\left(R_{k, i}^{b}\right)^{T}$. Unfortunately, the iterations do not result in this form. This is because in each iteration step $i$, except for the first iteration, in the right-hand side of (21), we have a different block cyclic matrix.

\subsection{Structure preserving solutions of PLDALEs}

The problem of preserving the block diagonal structure at the iterative computation of the Cholesky factor $\mathcal{R}_{i}$ can be circumvented by introducing a cyclic permutation matrix in each iteration step $i$ of (21). First, consider the trivial permutation matrix

$$
\Pi=\left[\begin{array}{ccccc}
J_{m_{0}} & 0 & \cdots & 0 & 0 \\
\vdots & J_{m_{1}} & 0 & & 0 \\
& & \ddots & & \vdots \\
0 & & & J_{m_{K-2}} & 0 \\
0 & 0 & \cdots & & J_{m_{K-1}}
\end{array}\right],
$$

where $J_{m_{i}}$ denotes the square identity matrix of size $m_{i}$, and $m_{i}$ equals the number of columns of $B_{i}$.

We introduce a new permutation matrix $\sigma^{i} \Pi$ at each iteration step $i$ in the computation of (20), where $\sigma^{i} \Pi$ changes in a cyclic manner by a forward block-column shift at each iteration step. 
For example, suppose that $K=3$, and $k=0,1,2$. Then for $i=1, \sigma^{0} \Pi=\Pi$ is given by

$$
\Pi=\left[\begin{array}{ccc}
J_{m_{0}} & 0 & 0 \\
0 & J_{m_{1}} & 0 \\
0 & 0 & J_{m_{2}}
\end{array}\right]
$$

For $i=2$, we get

$$
\sigma^{1} \Pi=\sigma \Pi=\sigma(\Pi)=\left[\begin{array}{ccc}
0 & J_{m_{0}} & 0 \\
0 & 0 & J_{m_{1}} \\
J_{m_{2}} & 0 & 0
\end{array}\right]
$$

which is just a forward shift of the last block-column of $\Pi$ in (23). Clearly for $i=3$, the permutation matrix takes the form

$$
\sigma^{2} \Pi=\sigma(\sigma \Pi)=\sigma(\sigma(\Pi))=\left[\begin{array}{ccc}
0 & 0 & J_{m_{0}} \\
J_{m_{1}} & 0 & 0 \\
0 & J_{m_{2}} & 0
\end{array}\right],
$$

which is nothing but a forward shift of the last block-column of $\sigma \Pi$. Note that similar types of permutations are also considered in [11] for solutions of periodic Lyapunov equations. One nice property of this permutation matrix is that it satisfies the periodicity property, i.e., $\sigma^{K} \Pi=\Pi$, where $K$ is the period of system (1).

Hence (20) takes the new form

$$
\mathcal{X}_{i}=\sum_{\ell=0}^{i-1}(\overline{\mathcal{E}} \mathcal{A})^{\ell} \mathcal{P}_{r} \overline{\mathcal{E}} \mathcal{B}\left(\sigma^{\ell} \Pi\right)\left(\sigma^{\ell} \Pi\right)^{\mathrm{T}} \mathcal{B}^{\mathrm{T}} \overline{\mathcal{E}}^{\mathrm{T}} \mathcal{P}_{r}^{\mathrm{T}}\left((\overline{\mathcal{E}} \mathcal{A})^{\mathrm{T}}\right)^{\ell}
$$

Therefore, in this new representation the Cholesky factor $\mathcal{R}_{i}$ has the form

$$
\mathcal{R}_{i}=\left[\mathcal{P}_{r} \overline{\mathcal{E}} \mathcal{B} \Pi,(\overline{\mathcal{E}} \mathcal{A}) \mathcal{P}_{r} \overline{\mathcal{E}} \mathcal{B}(\sigma \Pi), \ldots,(\overline{\mathcal{E}} \mathcal{A})^{i-1} \mathcal{P}_{r} \overline{\mathcal{E}} \mathcal{B}\left(\sigma^{i-1} \Pi\right)\right]
$$

In (26) we have $\mathcal{P}_{r} \overline{\mathcal{E}} \mathcal{B} \Pi=\mathcal{P}_{r} \overline{\mathcal{E}} \mathcal{B}$. It is to be noted that the matrix $\mathcal{R}_{i}$ in $(26)$ is not block diagonal but each block computed at the $i$-th iteration step of $\mathcal{R}_{i}$ in (26) preserves the block diagonal structure. Therefore, we construct the block diagonal Cholesky factor $\operatorname{diag}\left(R_{1, i}^{b}, \ldots, R_{K-1, i}^{b}, R_{0, i}^{b}\right)$ of $\mathcal{X}_{i}$ which is obtained from $\mathcal{R}_{i}$ by additional block permutation at each iteration step $i, i=2,3, \ldots$, and $X_{k}=R_{k} R_{k}^{T} \approx R_{k, i}^{b}\left(R_{k, i}^{b}\right)^{T}$ for $k=0,1, \ldots, K-1$. The whole iterative process is summarized in Algorithm 1 .

The approximate solution satisfies $\mathcal{R}_{i}=\mathcal{P}_{r} \mathcal{R}_{i}$ in every iteration step $i$. Algorithm 1 is to be stopped as soon as the normalized residual norm given by

$$
\eta\left(\mathcal{R}_{i}\right)=\frac{\left\|\mathcal{A} \mathcal{R}_{i} \mathcal{R}_{i}^{T} \mathcal{A}^{T}-\mathcal{E} \mathcal{R}_{i} \mathcal{R}_{i}^{T} \mathcal{E}^{T}+\mathcal{P}_{l} \mathcal{B} \mathcal{B}^{T} \mathcal{P}_{l}^{T}\right\|_{F}}{\left\|\mathcal{P}_{l} \mathcal{B} \mathcal{B}^{T} \mathcal{P}_{l}^{T}\right\|_{F}}
$$

satisfies the condition $\eta\left(\mathcal{R}_{i}\right)<t o l$ with a user-defined tolerance tol or a stagnation of residual norms is observed. 


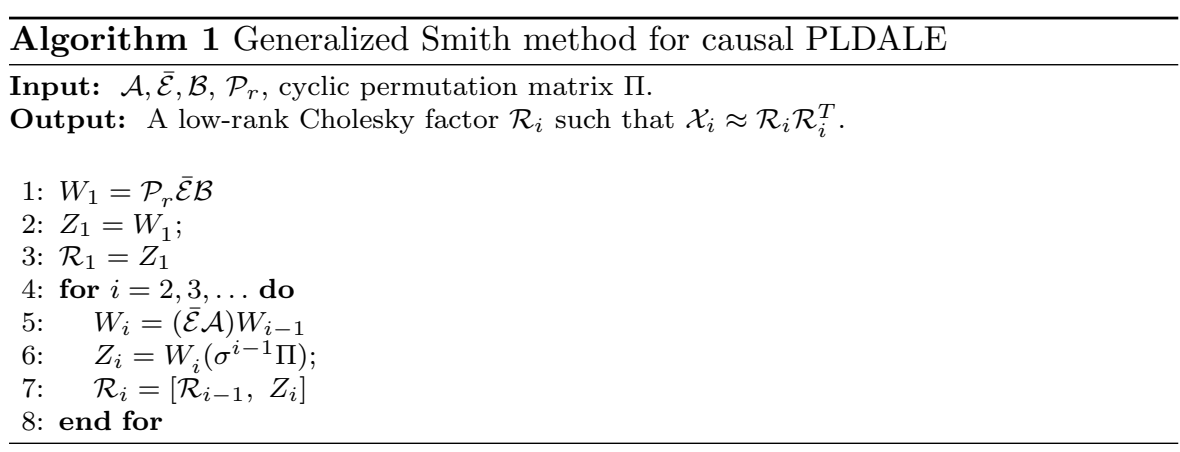

Remark 2 When the numbers of columns of $B_{k}$, i.e., $m_{k}, k=0,1, \ldots, K-1$, are big, $\mathcal{R}_{i}$ may face rank deficiency, because, in each iteration step, Algorithm 1 will add as many columns as there are in $\mathcal{B}$ to the previous $\mathcal{R}_{i}$. Hence, we propose the rank-revealing QR decomposition (RRQR) [8] of $\mathcal{R}_{i}$ with tolerance $\tau$ to compute a low-rank factor of $\mathcal{R}_{i}$. But in general, the RRQR will destroy the constructed block diagonal structure of $\mathcal{R}_{i}$. Hence, we use the RRQR decomposition on the factored form of $\mathcal{R}_{i}$, i.e., on $R_{k, i}^{b}$ at each iteration step $i$, for $k=0,1, \ldots, K-1$. Note that

$$
R_{k, i}^{b}=\left[R_{k, 1}, R_{k, 2}, \ldots, R_{k, i}\right]
$$

at the $i$-th iteration steps for $k=0,1, \ldots, K-1$. We then use the RRQR decomposition

$$
\left[\hat{V}_{i}, \hat{Q}_{i}, r_{c c}\right]=\operatorname{RRQR}\left(\left(R_{k, i}^{b}\right)^{T}, \tau_{c c}\right),
$$

where $\tau_{c c}=\sqrt{\epsilon}[2]$, update

$$
R_{k, i}^{b}=\hat{Q}_{i} \hat{V}_{i}^{T}\left[I_{r_{c c}}, 0\right]^{T},
$$

for $k=0,1, \ldots, K-1$, construct $\mathcal{R}_{i}=\operatorname{diag}\left(R_{1, i}^{b}, \ldots, R_{K-1, i}^{b}, R_{0, i}^{b}\right)$ at each iteration step $i$ in Step 7 of Algorithm 1, and use it in the $i+1$-st iteration step.

\subsection{Cyclic computation of causal PLDALEs}

In fact, the iteration (26) implemented in Algorithm 1 not only proves that each block matrix in $\mathcal{R}_{i}$ stay block diagonal at each iteration step $i$, it also enables us to rewrite (21) in such a way that one can directly compute the periodic Cholesky factors for different $k, k=0,1, \ldots, K-1$. From simple algebraic manipulation of (26), we observe that the periodic matrices $E_{k}, A_{k}$, and $B_{k}$ appear in a cyclic manner in the computation of the periodic Cholesky factors $R_{k, i}^{b}$ in every iteration step $i$ for different values of $k, k=0,1, \ldots, K-1$. Observing these cyclic relations and handing them technically, we can compute the periodic Cholesky factors $R_{k, i}^{b}, k=0,1, \ldots, K-1, i=1,2, \ldots$, directly. We represent some of those computations in the following. 
For $i=1$, we get

$$
\begin{aligned}
R_{0,1} & =R_{K, 1}=P_{r}(K) \bar{E}_{K-1} B_{K-1}=P_{r}(0) \bar{E}_{K-1} B_{K-1}, \\
R_{1,1} & =P_{r}(1) \bar{E}_{0} B_{0}, \quad \% \bar{E}_{K}=\bar{E}_{0}, B_{K}=B_{0} \\
& \vdots \\
R_{K-1,1} & =P_{r}(K-1) \bar{E}_{K-2} B_{K-2} .
\end{aligned}
$$

For $i=2$, we get

$$
\begin{aligned}
R_{0,2} & =R_{K, 2}=\bar{E}_{K-1} A_{K-1} P_{r}(K-1) \bar{E}_{K-2} B_{K-2}=\bar{E}_{K-1} A_{K-1} R_{K-1,1}, \\
R_{1,2} & =\bar{E}_{0} A_{0} P_{r}(0) \bar{E}_{K-1} B_{K-1}=\bar{E}_{0} A_{0} R_{0,1}, \\
& \vdots \\
R_{K-1,2} & =\bar{E}_{K-2} A_{K-2} P_{r}(K-2) \bar{E}_{K-3} B_{K-3}=\bar{E}_{K-2} A_{K-2} R_{K-2,1} .
\end{aligned}
$$

For $i=3$, we get

$$
\begin{aligned}
R_{0,3} & =R_{K, 3}=\bar{E}_{K-1} A_{K-1} \bar{E}_{K-2} A_{K-2} P_{r}(K-2) \bar{E}_{K-3} B_{K-3} \\
& =\bar{E}_{K-1} A_{K-1} R_{K-1,2}, \\
R_{1,3} & =\bar{E}_{0} A_{0} \bar{E}_{K-1} A_{K-1} P_{r}(K-1) \bar{E}_{K-2} B_{K-2}=\bar{E}_{0} A_{0} R_{0,2}, \\
& \vdots \\
R_{K-1,3} & =\bar{E}_{K-2} A_{K-2} \bar{E}_{K-3} A_{K-3} P_{r}(K-3) \bar{E}_{K-4} B_{K-4} \\
& =\bar{E}_{K-2} A_{K-2} R_{K-2,2},
\end{aligned}
$$

and so on. The whole computation is summarized in Algorithm 2. Note that in the above computations and also in Algorithm 2, we use the periodicity of the coefficient matrices and that of the projectors. Here, $P_{r}(K)=P_{r}(0)$, $P_{r}(K-1)=P_{r}(-1), \bar{E}_{K}=\bar{E}_{0}, \bar{E}_{-1}=\bar{E}_{K-1}, \bar{E}_{K+k}=\bar{E}_{k}$, and the similar for others. Obviously, $X_{K}=X_{0}=R_{K} R_{K}^{T}=R_{0} R_{0}^{T}$. It should be also noted that in Algorithm 2, $R_{k, i}^{b}$ means the computed $R_{k}$ at the $i$-th iteration steps. Also note that $R_{k, i}^{b}$ collects all these iterative counterparts for an individual $k$, where $k=0,1, \ldots, K-1$. That means for $k=0$, we compute $R_{0, i}^{b}=$ $\left[R_{0,1}, R_{0,2}, \ldots, R_{0, i}\right]$, and similarly the others. 


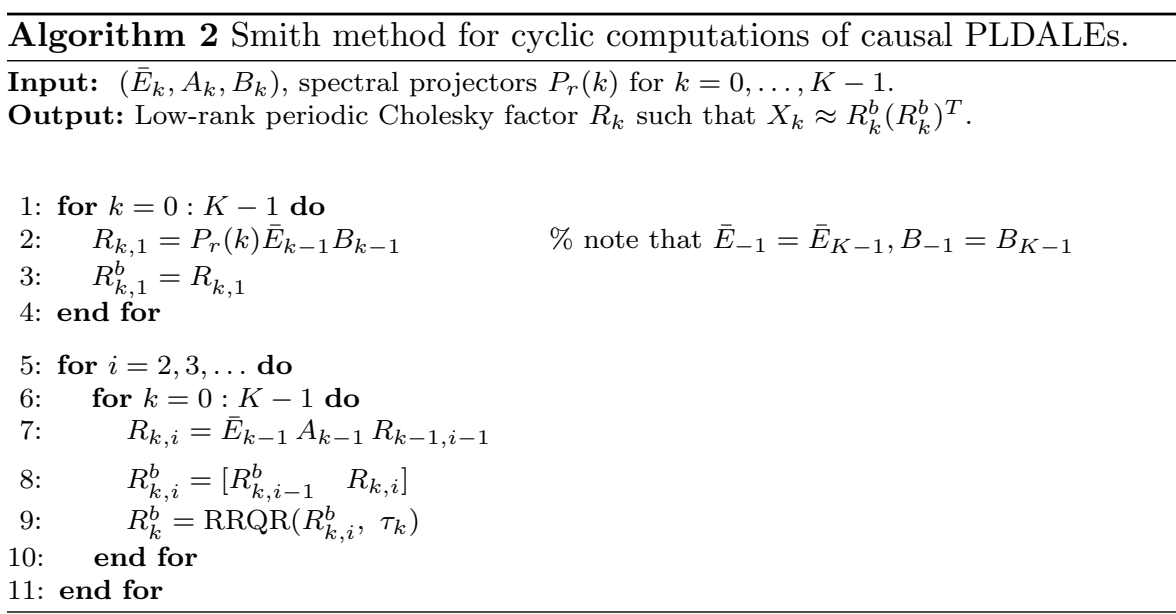

One can define a stoping criteria for Algorithm 2 by setting a normalized residual norm given by

$$
\eta\left(R_{k}^{b}\right)=\frac{\left\|A_{k} R_{k}^{b}\left(R_{k}^{b}\right)^{T} A_{k}^{T}-E_{k} R_{k+1}^{b}\left(R_{k+1}^{b}\right)^{T} E_{k}^{T}+P_{l}(k) B_{k} B_{k}^{T} P_{l}(k)^{T}\right\|_{F}}{\left\|P_{l}(k) B_{k} B_{k}^{T} P_{l}(k)^{T}\right\|_{F}}
$$

which satisfy the condition $\eta\left(R_{k}^{b}\right)<t_{0} l_{c}$ for $k=0,1, \ldots, K-1$, where $t_{0} l_{c}$ is a user predefined tolerance. A similar computation can also be stated for the causal periodic observability Gramians $Y_{k}$ of (9).

\section{Smith method for noncausal PLDALEs}

For nonsingular $\mathcal{A}$, the PLDALE (12) is equivalent to the PLDALE

$$
\begin{aligned}
\hat{\mathcal{X}}-\left(\mathcal{A}^{-1} \mathcal{E}\right) \hat{\mathcal{X}}\left(\mathcal{A}^{-1} \mathcal{E}\right)^{T} & =\mathcal{Q}_{r} \mathcal{A}^{-1} \mathcal{B B}^{T} \mathcal{A}^{-T} \mathcal{Q}_{r}^{T}, \\
\hat{\mathcal{X}} & =\mathcal{Q}_{r} \hat{\mathcal{X}} \mathcal{Q}_{r}^{T} .
\end{aligned}
$$

Such an equation can be solved by the Smith method [21] given by

$$
\begin{aligned}
& \hat{\mathcal{X}}_{1}=\mathcal{Q}_{r} \mathcal{A}^{-1} \mathcal{B B}^{T} \mathcal{A}^{-T} \mathcal{Q}_{r}^{T}, \\
& \hat{\mathcal{X}}_{i}=\mathcal{Q}_{r} \mathcal{A}^{-1} \mathcal{B B}^{T} \mathcal{A}^{-T} \mathcal{Q}_{r}^{T}+\left(\mathcal{A}^{-1} \mathcal{E}\right) \hat{\mathcal{X}}_{i-1}\left(\mathcal{A}^{-1} \mathcal{E}\right)^{T} .
\end{aligned}
$$

Note that in the noncausal case we do not need to compute the reflexive generalized inverses of the periodic matrices $E_{k}$, and $A_{k}$, for $k=0,1, \ldots, K-1$, since (31) requires only the inversion of the cyclic lifted nonsingular matrix $\mathcal{A}$.

In this case, $\mathcal{Q}_{r} \mathcal{A}^{-1} \mathcal{E}=\mathcal{A}^{-1} \mathcal{E} \mathcal{Q}_{r}$ is nilpotent with the nilpotency index $\nu$ [2], and after $\nu$ iterations we obtain

$$
\hat{\mathcal{X}}_{\nu}=\sum_{i=0}^{\nu-1}\left(\mathcal{A}^{-1} \mathcal{E}\right)^{i} \mathcal{Q}_{r} \mathcal{A}^{-1} \mathcal{B B}^{T} \mathcal{A}^{-T} \mathcal{Q}_{r}^{T}\left(\left(\mathcal{A}^{-1} \mathcal{E}\right)^{T}\right)^{i}=\hat{\mathcal{X}}
$$


Therefore, the Cholesky factor $\hat{\mathcal{R}}$ of the solution $\hat{\mathcal{X}}=\hat{\mathcal{R}} \hat{\mathcal{R}}^{T}$ of (30) and also of the PLDALE (12) takes the form

$$
\hat{\mathcal{R}}_{\nu}=\left[\mathcal{Q}_{r} \mathcal{A}^{-1} \mathcal{B}, \mathcal{A}^{-1} \mathcal{E} \mathcal{Q}_{r} \mathcal{A}^{-1} \mathcal{B}, \ldots,\left(\mathcal{A}^{-1} \mathcal{E}\right)^{\nu-1} \mathcal{Q}_{r} \mathcal{A}^{-1} \mathcal{B}\right]
$$

Note that the above iteration does not preserve the block diagonal structure at every iteration step in the computation of the Cholesky factor $\hat{\mathcal{R}}_{\nu}$ [2]. By introducing a cyclic permutation matrix in each iteration step like the causal case, one can easily preserve the block diagonal structure at every iteration step in(33).

6.1 Structure preserving solutions for noncausal PLDALEs

Let us again consider a cyclic permutation matrix $\hat{\mathcal{P}}$ of the form

$$
\hat{\mathcal{P}}=\left[\begin{array}{ccccc}
0 & & \cdots & 0 & J_{m_{0}} \\
J_{m_{1}} & & & & 0 \\
& \ddots & & & \vdots \\
0 & & & J_{m_{K-1}} & 0
\end{array}\right]
$$

where as before, $J_{m_{i}}$ denotes the identity matrix of size $m_{i}$. We introduce a new permutation matrix $\hat{\sigma}^{i} \hat{\mathcal{P}}$ at each iteration step $i$ in the computation of (32), where $\hat{\sigma}^{i} \hat{\mathcal{P}}$ changes in a cyclic manner by a backward block-column shift at each iteration step.

Similar to the causal case, the permutation matrix is periodic with a periodicity $K$, i.e., $\hat{\sigma}^{K} \hat{\mathcal{P}}=\hat{\mathcal{P}}$, where $K$ is the period of system (1). Then (32) gets the new form

$$
\hat{\mathcal{X}}_{\nu}=\sum_{i=0}^{\nu-1}\left(\mathcal{A}^{-1} \mathcal{E}\right)^{i} \mathcal{Q}_{r} \mathcal{A}^{-1} \mathcal{B}\left(\hat{\sigma}^{i} \hat{\mathcal{P}}\right)\left(\hat{\sigma}^{i} \hat{\mathcal{P}}\right)^{T} \mathcal{B}^{T} \mathcal{A}^{-T} \mathcal{Q}_{r}^{T}\left(\left(\mathcal{A}^{-1} \mathcal{E}\right)^{T}\right)^{i}=\hat{\mathcal{X}}
$$

Therefore, the Cholesky factor $\hat{\mathcal{R}}_{\nu}$ has the form

$$
\hat{\mathcal{R}}_{\nu}=\left[\mathcal{Q}_{r} \mathcal{A}^{-1} \mathcal{B} \hat{\mathcal{P}}, \mathcal{A}^{-1} \mathcal{E} \mathcal{Q}_{r} \mathcal{A}^{-1} \mathcal{B}(\hat{\sigma} \hat{\mathcal{P}}), \ldots,\left(\mathcal{A}^{-1} \mathcal{E}\right)^{\nu-1} \mathcal{Q}_{r} \mathcal{A}^{-1} \mathcal{B}\left(\hat{\sigma}^{\nu-1} \hat{\mathcal{P}}\right)\right]
$$

It can be verified that each column block in (36) preserves the block diagonal structure analogous to the solution of (30). The computation of this factor is presented in Algorithm 3.

We note that if the index $\nu$ is unknown, then Algorithm 3 can be stopped as soon as $\left\|W_{i}\right\|_{F} \leq \epsilon$ or $\left\|W_{i}\right\|_{F} /\left\|\hat{\mathcal{R}}_{i}\right\|_{F} \leq \epsilon$ with the machine precision $\epsilon$. Thus, for systems of low index, the solution of (8) can be obtained with few computations. Also, we have to project $W_{i}$ onto the image of $\mathcal{Q}_{r}$ to guarantee that the second equation in (12) (and also in (30)) is satisfied. 


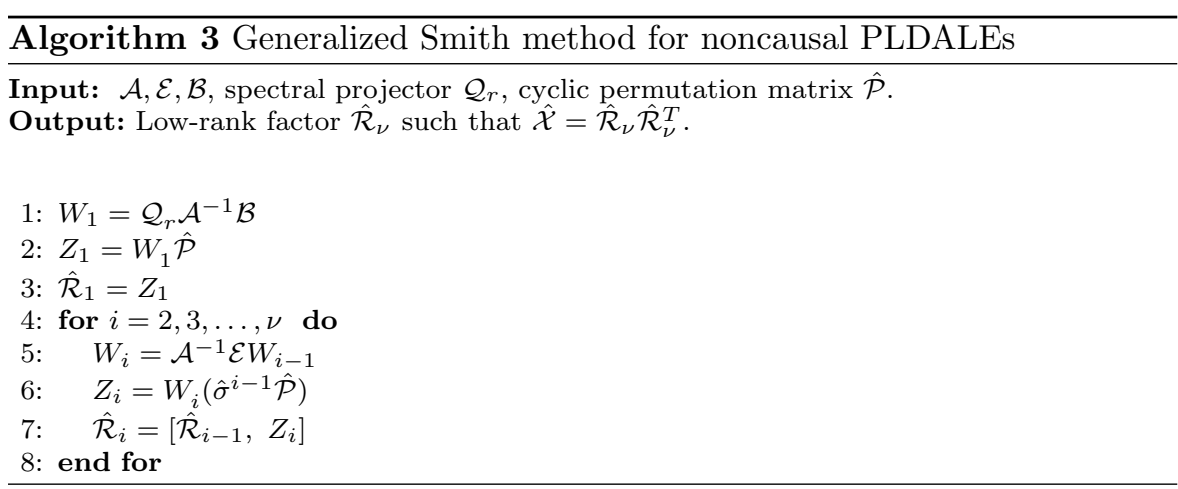

Note that the matrix $\hat{\mathcal{R}}_{\nu}$ in (36) is not block diagonal but each column block computed at the $i$-th iteration step in (36) preserves the block diagonal structure analogous to the solution of (30). Therefore, we construct the block diagonal Cholesky factor $\operatorname{diag}\left(\hat{R}_{1, i}^{b}, \ldots, \hat{R}_{K-1, i}^{b}, \hat{R}_{0, i}^{b}\right)$ of $\hat{\mathcal{X}}_{i}$ which is obtained from $\hat{\mathcal{R}}_{i}$ by additional block permutation at each iteration step $i, i=2,3, \ldots, \nu$. Note that $\hat{R}_{k, i}^{b}$ collects all the iterative counterparts of the computed $\hat{R}_{k}$ at the $i$-th iteration steps for $k=0,1, \ldots, K-1$, and $\hat{X}_{k}=\hat{R}_{k} \hat{R}_{k}^{T} \approx \hat{R}_{k, i}^{b}\left(\hat{R}_{k, i}^{b}\right)^{T}$.

\subsection{Cyclic computations of periodic noncausal Cholesky factors}

In fact, the iteration (36) implemented in Algorithm 3 not only proves that each column block computed in $\hat{\mathcal{R}}_{\nu}$ stay block diagonal, it also enables us to rewrite (33) in such a way that one can directly compute the periodic Cholesky factors for different $k, k=0,1, \ldots, K-1$. From simple algebraic manipulation of (36), we observe that the periodic matrices $E_{k}, A_{k}$, and $B_{k}$ appear in a cyclic manner in the computation of the periodic Cholesky factors $\hat{R}_{k, i}^{b}$ in every iteration step $i$ for different values of $k, k=0,1, \ldots, K-1$. Observing these cyclic relations and handing them technically, we can compute the periodic Cholesky factors $\hat{R}_{k, i}^{b}, k=0,1, \ldots, K-1, i=1,2, \ldots$, directly. We represent some of those computations in the following.

For $i=1$, we get

$$
\begin{aligned}
\hat{R}_{0,1} & =\hat{R}_{K, 1}=Q_{r}(0) A_{0}^{-1} B_{0}, \\
\hat{R}_{1,1} & =Q_{r}(1) A_{1}^{-1} B_{1}, \\
& \vdots \\
\hat{R}_{K-1,1} & =Q_{r}(K-1) A_{K-1}^{-1} B_{K-1} .
\end{aligned}
$$


For $i=2$, we get

$$
\begin{aligned}
\hat{R}_{0,2} & =\hat{R}_{K, 2}=A_{0}^{-1} E_{0} Q_{r}(1) A_{1}^{-1} B_{1}=A_{0}^{-1} E_{0} \hat{R}_{1,1}, \\
\hat{R}_{1,2} & =A_{1}^{-1} E_{1} Q_{r}(2) A_{2}^{-1} B_{2}=A_{1}^{-1} E_{1} \hat{R}_{2,1} \\
& \vdots \\
\hat{R}_{K-1,2} & =A_{K-1}^{-1} E_{K-1} Q_{r}(0) A_{0}^{-1} B_{0}=A_{K-1}^{-1} E_{K-1} \hat{R}_{0,1} .
\end{aligned}
$$

For $i=3$, we get

$$
\begin{aligned}
\hat{R}_{0,3} & =\hat{R}_{K, 3}=A_{0}^{-1} E_{0} A_{1}^{-1} E_{1} Q_{r}(2) A_{2}^{-1} B_{2}=A_{0}^{-1} E_{0} \hat{R}_{1,2}, \\
\hat{R}_{1,3} & =A_{1}^{-1} E_{1} A_{2}^{-1} E_{2} Q_{r}(3) A_{3}^{-1} B_{3}=A_{1}^{-1} E_{1} \hat{R}_{2,2}, \\
& \vdots \\
\hat{R}_{K-1,3} & =A_{K-1}^{-1} E_{K-1} A_{0}^{-1} E_{0} Q_{r}(1) A_{1}^{-1} B_{1}=A_{K-1}^{-1} E_{K-1} R_{0,2},
\end{aligned}
$$

and so on.

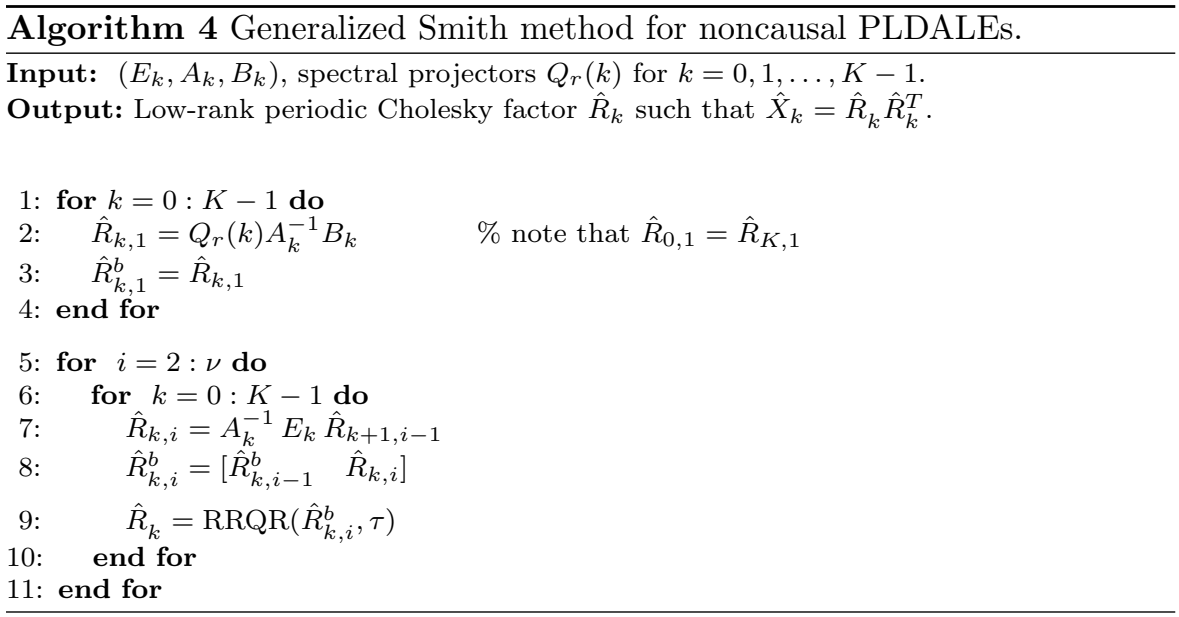

The whole computation is summarized in Algorithm 4. Similar to the causal case, we consider the periodicity of the coefficient matrices and that of the projectors in Algorithm 4. Here $Q_{r}(K)=Q_{r}(0), E_{K}=E_{0}, E_{K-1}=E_{-1}, E_{K+k}=$ $E_{k}$, etc. Obviously, $\hat{X}_{K}=\hat{X}_{0}=\hat{R}_{K} \hat{R}_{K}^{T}=\hat{R}_{0} \hat{R}_{0}^{T}$. In Algorithm $4, \hat{R}_{k, i}^{b}$ means the computed $\hat{R}_{k}$ at the $i$-th iteration step. Also note that $\hat{R}_{k, i}^{b}$ collocates all these iterative counterparts for an individual $k$, where $k=0,1, \ldots, K-1$. That means for $k=0$, we compute

$$
\hat{R}_{0, i}^{b}=\left[\hat{R}_{0,1}, \hat{R}_{0,2}, \ldots, \hat{R}_{0, i}\right]
$$


and similarly the others.

For unknown index $\nu$, we propose the RRQR decomposition [8] of $\hat{R}_{k, i}^{b}$ with tolerance $\tau$ to truncate redundant columns in the iterations of Algorithm 4. In that case, we assume that after the $i$-th iteration we have the exact computation of the periodic Cholesky factors $\hat{R}_{k}$ satisfying relation $\left\|\hat{R}_{k, i}\right\|_{F} \leq \epsilon$ or $\left\|\hat{R}_{k, i}\right\|_{F} /\left\|\hat{R}_{k}\right\|_{F} \leq \epsilon$, where $\epsilon$ is the machine precision, and then, $\hat{X}_{k}=\hat{R}_{k} \hat{R}_{k}^{T}$ are the periodic solutions of (8) for $k=0,1, \ldots, K-1$. We also need to project $\hat{R}_{k, i}$ onto the image of $Q_{r}$ to guarantee that the second equation in (8) is satisfied.

Remark 3 As the $A_{k}$ can be singular in the discrete-time case, Algorithm 4 is restricted in that case. We restrict our model reduction approach for systems where $A_{k}$, for $k=0,1, \ldots, K-1$, are nonsingular.

Remark 4 The causal and noncausal observabiliy Gramians of the periodic descriptor system (1) can also be determined from the corresponding PLDALEs that are dual to the PLDALE (11) and (12), see [1] for details. Applying Algorithm 1 and Algorithm 3 (and also their corresponding cyclic reformulations Algorithm 2 and Algorithm 4) to these equations, we find, respectively, the lowrank Cholesky factors $L_{k}$ of the causal observability Gramians $Y_{k} \approx L_{k} L_{k}^{T}$ and the Cholesky factor $\tilde{Y}_{k}$ of the noncausal observability Gramians $\hat{Y}_{k}=\hat{L}_{k} \hat{L}_{k}^{T}$.

\section{Application to model order reduction}

Model order reduction (MOR) is an approach, where a large dynamical system is approximated by a reduced-order model which can be fast and efficiently simulated and which has nearly the same response characteristics as compared to the original large model. Balanced truncation for periodic standard discretetime system and periodic descriptor systems has been considered in $[7,30]$, and [6], respectively. For the periodic descriptor system (1), a reduced-order model of dimension $\overline{\mathbf{r}}=\left(r_{0}, r_{1}, \ldots, r_{K-1}\right)$ has the form

$$
\tilde{E}_{k} \tilde{x}_{k+1}=\tilde{A}_{k} \tilde{x}_{k}+\tilde{B}_{k} u_{k}, \quad \tilde{y}_{k}=\tilde{C}_{k} \tilde{x}_{k}, \quad k \in \mathbb{Z},
$$

where $\tilde{E}_{k} \in \mathbb{R}^{\gamma_{k} \times r_{k+1}}, \tilde{A}_{k} \in \mathbb{R}^{\gamma_{k} \times r_{k}}, \tilde{B}_{k} \in \mathbb{R}^{\gamma_{k} \times m_{k}}, \tilde{C}_{k} \in \mathbb{R}^{q_{k} \times r_{k}}$ are $K$ periodic matrices, $\sum_{k=0}^{K-1} \gamma_{k}=\sum_{k=0}^{K-1} r_{k}=\mathbf{r}$, and $\mathbf{r} \ll \mathbf{n}$. It is also important that the reduced-order model preserves physical properties of the original system such as regularity and stability, and that the approximation error is small.

Consider that the set of periodic matrix pairs $\left\{\left(E_{k}, A_{k}\right)\right\}_{k=0}^{K-1}$ is periodic stable, and the Cholesky factors of the causal and noncausal Gramians satisfy

$$
\begin{array}{ll}
X_{k}=R_{k} R_{k}^{T}, & Y_{k}=L_{k} L_{k}^{T}, \\
\hat{X}_{k}=\hat{R}_{k} \hat{R}_{k}^{T}, & \hat{Y}_{k}=\hat{L}_{k} \hat{L}_{k}^{T} .
\end{array}
$$

Then the causal and noncausal Hankel singular values of the periodic descriptor system (1) are defined as

$$
\sigma_{k, j}=\zeta_{j}\left(L_{k}^{T} E_{k-1} R_{k}\right), \quad \theta_{k, j}=\zeta_{j}\left(\hat{L}_{k+1}^{T} A_{k} \hat{R}_{k}\right),
$$


respectively, where $\zeta_{j}($.$) denotes the singular values of the corresponding prod-$ uct matrices. For a balanced system, truncation of states related to the small causal Hankel singular values does not change system properties essentially. Unfortunately, truncation of small non-zero noncausal Hankel singular values may lead the system to become unstable [9].

Let us consider the singular value decompositions of the product matrices

$$
\begin{aligned}
L_{k}^{T} E_{k-1} R_{k} & =\left[U_{k, 1}, U_{k, 2}\right]\left[\begin{array}{ll}
\Sigma_{k, 1} & \\
& \Sigma_{k, 2}
\end{array}\right]\left[V_{k, 1}, V_{k, 2}\right]^{T}, \\
& \hat{L}_{k+1}^{T} A_{k} \hat{R}_{k}=U_{k, 3} \Theta_{k} V_{k, 3}^{T},
\end{aligned}
$$

where $\left[U_{k, 1}, U_{k, 2}\right],\left[V_{k, 1}, V_{k, 2}\right], U_{k, 3}$ and $V_{k, 3}$ are orthogonal,

$$
\Sigma_{k, 1}=\operatorname{diag}\left(\sigma_{k, 1}, \ldots, \sigma_{k, r_{k}^{f}}\right), \quad \Sigma_{k, 2}=\operatorname{diag}\left(\sigma_{k, r_{k}^{f}+1}, \ldots, \sigma_{k, n_{k}^{f}}\right),
$$

with $\sigma_{k, 1} \geq \cdots \geq \sigma_{k, r_{k}^{f}}>\sigma_{k, r_{k}^{f}+1} \geq \ldots \geq \sigma_{k, n_{k}^{f}}$, and $\Theta_{k}=\operatorname{diag}\left(\theta_{k, 1}, \ldots, \theta_{k, r_{k}^{\infty}}\right)$ is nonsingular for $k=0,1, \ldots, K-1$.

Defining the projection matrices $[6]$ as

$$
\begin{aligned}
S_{k, r} & =\left[L_{k+1} U_{k+1,1} \Sigma_{k+1,1}^{-1 / 2}, \quad \hat{L}_{k+1} U_{k, 3} \Theta_{k}^{-1 / 2}\right] \in \mathbb{R}^{\mu_{k} \times \gamma_{k+1}}, \\
T_{k, r} & =\left[R_{k} V_{k, 1} \Sigma_{k, 1}^{-1 / 2}, \quad \hat{R}_{k} V_{k, 3} \Theta_{k}^{-1 / 2}\right] \in \mathbb{R}^{n_{k} \times r_{k}}
\end{aligned}
$$

with $r_{k}=r_{k}^{f}+r_{k}^{\infty}$ and $\gamma_{k+1}=r_{k+1}^{f}+r_{k}^{\infty}$, we compute the reduced-order system (37) as

$$
\tilde{E}_{k}=S_{k, r}^{T} E_{k} T_{k+1, r}, \tilde{A}_{k}=S_{k, r}^{T} A_{k} T_{k, r}, \tilde{B}_{k}=S_{k, r}^{T} B_{k}, \tilde{C}_{k}=C_{k} T_{k, r},
$$

Let $\tilde{\mathcal{H}}(z)$ be the transfer functions of the reduced-order lifted system formed from the reduced-order matrices in (39). Then the reduced-order model satisfies the following $\mathbb{H}_{\infty}$-norm error bound

$$
\begin{aligned}
\|\mathcal{H}-\tilde{\mathcal{H}}\|_{\mathbb{H}_{\infty}} & =\sup _{\omega \in[0,2 \pi]}\left\|\mathcal{H}\left(e^{i \omega}\right)-\tilde{\mathcal{H}}\left(e^{i \omega}\right)\right\|_{2} \\
& \leq 2 \operatorname{trace}\left(\operatorname{diag}\left(\Sigma_{0,2}, \ldots, \Sigma_{K-1,2}\right)\right),
\end{aligned}
$$

where $\mathcal{H}(z)$ is defined in (4), $\|.\|_{2}$ denotes the spectral matrix norm, and $\Sigma_{k, 2}$, $k=0,1, \ldots, K-1$, contains the truncated causal Hankel singular values $[9$, 2].

\section{Results}

Example 1 We consider first an artificial periodic discrete-time descriptor system on index-1 from [2, Example 1], which is reformulated from its original model in [6, Example 1]. In this reformulation, the periodic descriptor system has $\mu_{k}=n_{k}=404, m_{k}=2$ and $p_{k}=3$ for the periodicity $K=10$, i.e., $k=0,1, \ldots, 9$. The spectral projectors $P_{l}(k)$ and $P_{r}(k)$ are computed using the Kronecker-like forms of periodic matrix pairs $\left\{\left(E_{k}, A_{k}\right)\right\}_{k=0}^{K-1}$ proposed 


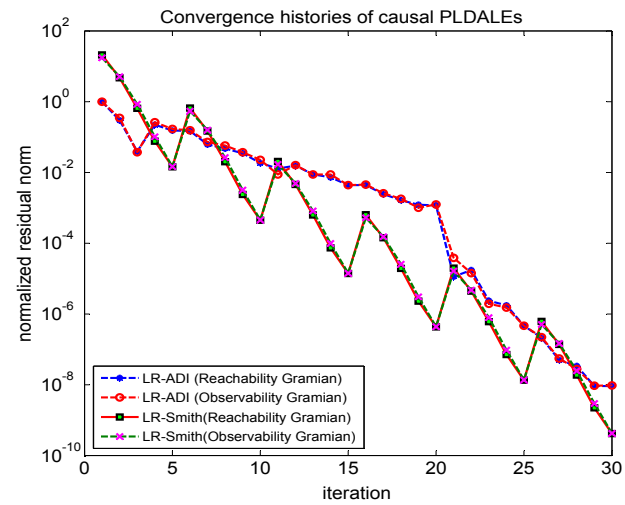

(a)

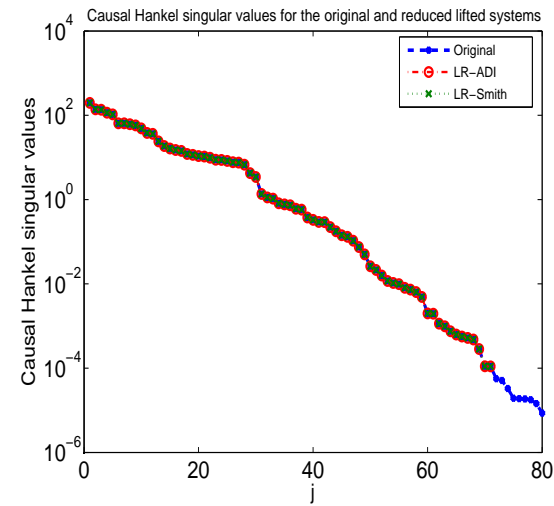

(b)

Fig. 1 (a) Normalized residual norms for the causal reachability and observability lifted projected Lyapunov equations; (b) Causal Hankel singular values for original and reducedorder lifted systems.

in $[28,6]$. The set of periodic matrix pairs $\left\{\left(E_{k}, A_{k}\right)\right\}_{k=0}^{K-1}$ is periodic stable with causal eigenvalues, $n_{k}^{f}=400$ and noncausal eigenvalues, $n_{k}^{\infty}=4$ for $k=0,1, \ldots, 9$. The original lifted system has order $\mathbf{n}=4040$. Since $n_{k}^{f}=400$ for $k=0,1, \ldots, 9$, computing the $\bar{E}_{k}$ is straightforward using relation (15).

We solve the causal and noncausal lifted projected Lyapunov equations using Algorithm 2 and Algorithm 4, respectively. Since, Algorithm 2 is the cyclic reformulation of Algorithm 1, we compute the normalized residual norms at each Smith iteration step for the reachability and observability type of the causal lifted Lyapunov equations using relation (27). We apply the RRQR decomposition at every fifth iteration step, since it is computationally expensive. Experiments confirm that computing it every $\ell=5$ steps often provides a good efficiency balance of computational cost and rank growth compared to other choices of $\ell$. To illustrate the efficiencies of the proposed algorithms, we compare the results with the corresponding ADI (Alternating Direction Implicit) computation of [2]. Fig. 1(a) shows the decay of the residual norms computed at each Smith iteration step.

This iteration is stopped as soon as the normalized Lyapunov residual reaches the tolerance $t o l=10^{-10}$. We approximate system (1) by a reducedorder model obtained by truncating the states corresponding to the small causal Hankel singular values satisfying $\sigma_{k, j}<10^{-4}$. The largest 80 causal Hankel singular values of the original lifted system, and the approximate 71 causal Hankel singular values for the reduced-order lifted system are shown in Fig. 1(b).

For different subsystems, the numbers of the computed non-zero noncausal Hankel singular values are identical and given by $r_{k}^{\infty}=2$ for $k=0,1, \ldots, 9$. The computed reduced-order model has subsystems of orders $(9,9,9,9,9,9,9,9$, $10,9)$. Note that stability is preserved in the reduced-order system. 


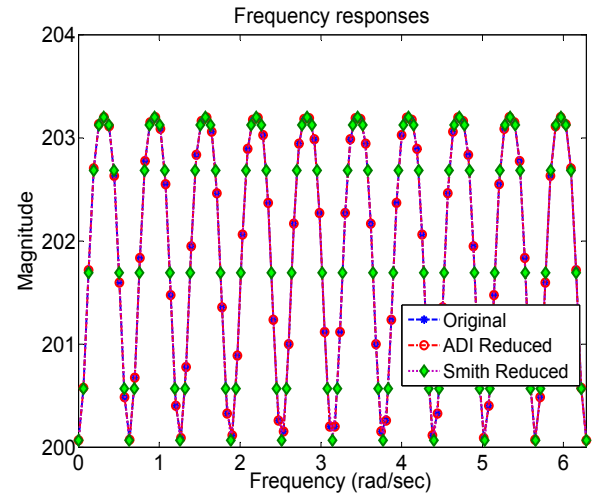

(a)

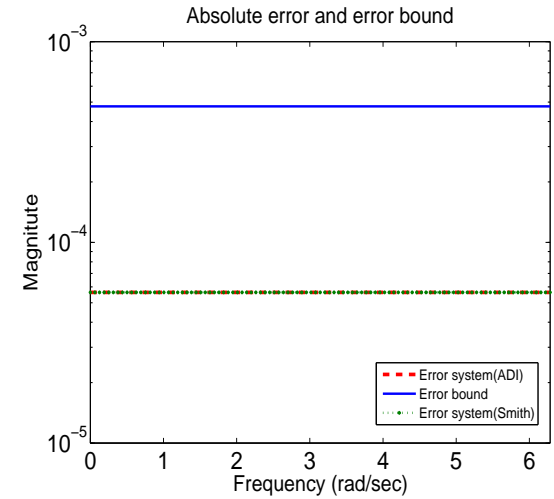

(b)

Fig. 2 (a) The frequency responses of the original and the reduced-order lifted systems; (b) absolute error and error bound.

Fig. 2(a) shows the norms of the frequency responses $\mathcal{H}\left(e^{i \omega}\right)$ and $\tilde{\mathcal{H}}\left(e^{i \omega}\right)$ of the original and reduced-order lifted systems for a frequency range $[0,2 \pi]$. We observe a good match of the system norms. Finally, in Fig. 2(b), we display the absolute error $\left\|\mathcal{H}\left(e^{i \omega}\right)-\tilde{\mathcal{H}}\left(e^{i \omega}\right)\right\|_{2}$ and the error bound given in (40).

Example 2 As a second model problem we consider here an artificial continuoustime model from Section 4.3 of [26], where a spring-damper model is considered as an artificial model of piezo-mechanical systems. We consider $n=500, l=$ 100 , nin $=2$, nout $=3$ for our model problem, and hence the dimension of the continuous-time model is $2 n+l=1100$. The formulated continuous-time model is converted to a discrete-time model by an Euler discretization method [20]. We then change the damping matrix periodically by introducing some periodic coefficients inside it. As a result, the model is time-varying and periodic. The details of this periodic model formulation are given in the appendix.

For the resulting periodic model, we have $n_{k}=1100, m_{k}=2, p_{k}=3$, and a period $K=10$. The periodic matrix pairs $\left\{\left(E_{k}, A_{k}\right)\right\}_{k=0}^{K-1}$ are periodic stable with $n_{k}^{f}=1000$ and $n_{k}^{\infty}=100$ for every $k=0,1, \ldots, 9$. The resulting periodic system is of index 1 , and the original lifted system has order $\mathbf{n}=11000$. The sparsity pattern of the periodic pair at $k=0$ is shown in Fig. 3 .

In Fig. 4(a), we present the largest 260 causal Hankel singular values computed by the proposed Smith method in Algorithm 2. We approximate system (1) to the tolerance $10^{-4}$ and truncate the states corresponding to the smallest 200 causal Hankel singular values. The system has 20 noncausal Hankel singular values which are positive, but very small. The values of these noncausal Hankel singular values lie in the range of $\left[10^{-13}, 10^{-15}\right]$, and they are shown in Fig. 4(b). It is to be mentioned that our model problem is of index 1, and hence we need only one iteration to compute the noncausal Cholesky factor for noncausal PLDALEs using Equation (36). 

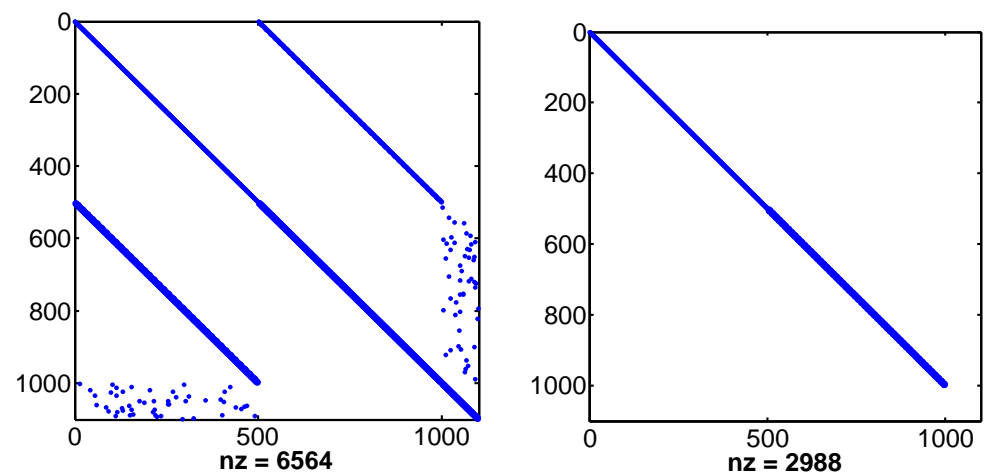

Fig. 3 Sparsity patterns of $A_{0}$ (left) and $E_{0}$ (right).

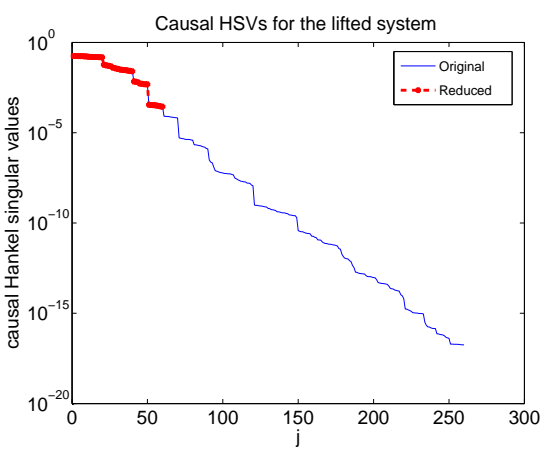

(a)

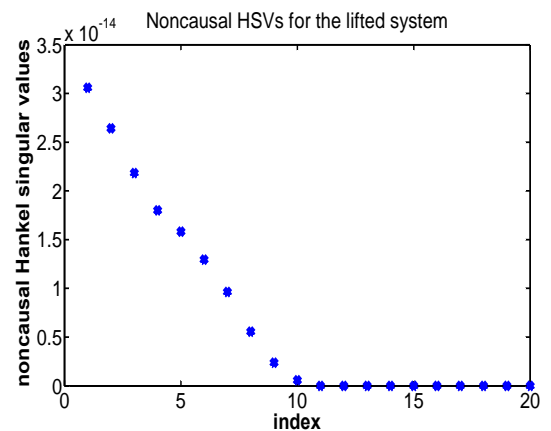

(b)

Fig. 4 (a) Causal Hankel singular values for original and reduced-order lifted systems. (b) Noncausal Hankel singular values for original and reduced-order lifted systems.

The computed reduced-order model has subsystems of order $\overline{\mathbf{r}}=(9,8,8,7,8$, $9,7,8,8,8)$, and $\mathbf{r}=80$. In Fig. $5(\mathrm{a})$, we show the norms of the frequency responses $\mathcal{H}$ and $\tilde{\mathcal{H}}$ for a frequency range $[0,2 \pi]$. We also display the absolute error $\left\|\mathcal{H}\left(e^{i \omega}\right)-\tilde{\mathcal{H}}\left(e^{i \omega}\right)\right\|_{2}$ and the error bound in Fig. 5(b). One can see that the error bound is tight in this example.

\section{Conclusions}

We discussed the structure preserving Smith iterations to compute the lowrank factors for the solutions of large sparse projected periodic discrete-time algebraic Lyapunov equations exploiting the reflexive generalized inverses of the periodic matrix coefficients associated with the periodic descriptor system. These low-rank factors are used in a balanced truncation model reduction approach to find a reduced-order model for periodic discrete-time descriptor systems. The proposed model reduction method delivers a reduced-order model that preserves the regularity and stability properties of the original system. 

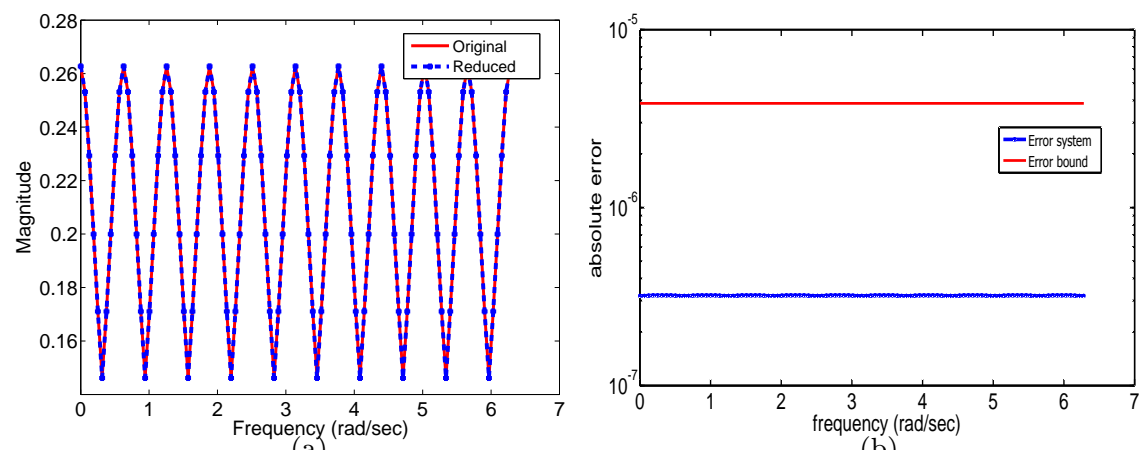

(a)

(b)

Fig. 5 The frequency responses and error bounds of the original and the reduced-order lifted systems.

An important advantage of our computational approach is that one can directly compute the reflexive generalized inverses of the periodic descriptor system, without explicitly manipulating the lifted representations. Beside this, the proposed Smith iterations preserve the cyclic block diagonal structures at all iteration steps which is the main challenging task in periodic iterative computations.

The major drawback of our proposed method is that it requires the periodic projectors explicitly for the computations of periodic reflexive generalized inverses which is numerically expensive and not a wise approach for higher index systems. Hence we restrict the proposed model reduction approach for systems of index-1. 
Appendix

The MATLAB codes of the periodic model formulation used in the second model problem. The following codes will construct the periodic matrices $E_{k}$, $A_{k}, B_{k}$, and $C_{k}$ for $k=0,1, \ldots, 9$. Note that $E_{k}$ are constant matrices in the following construction.

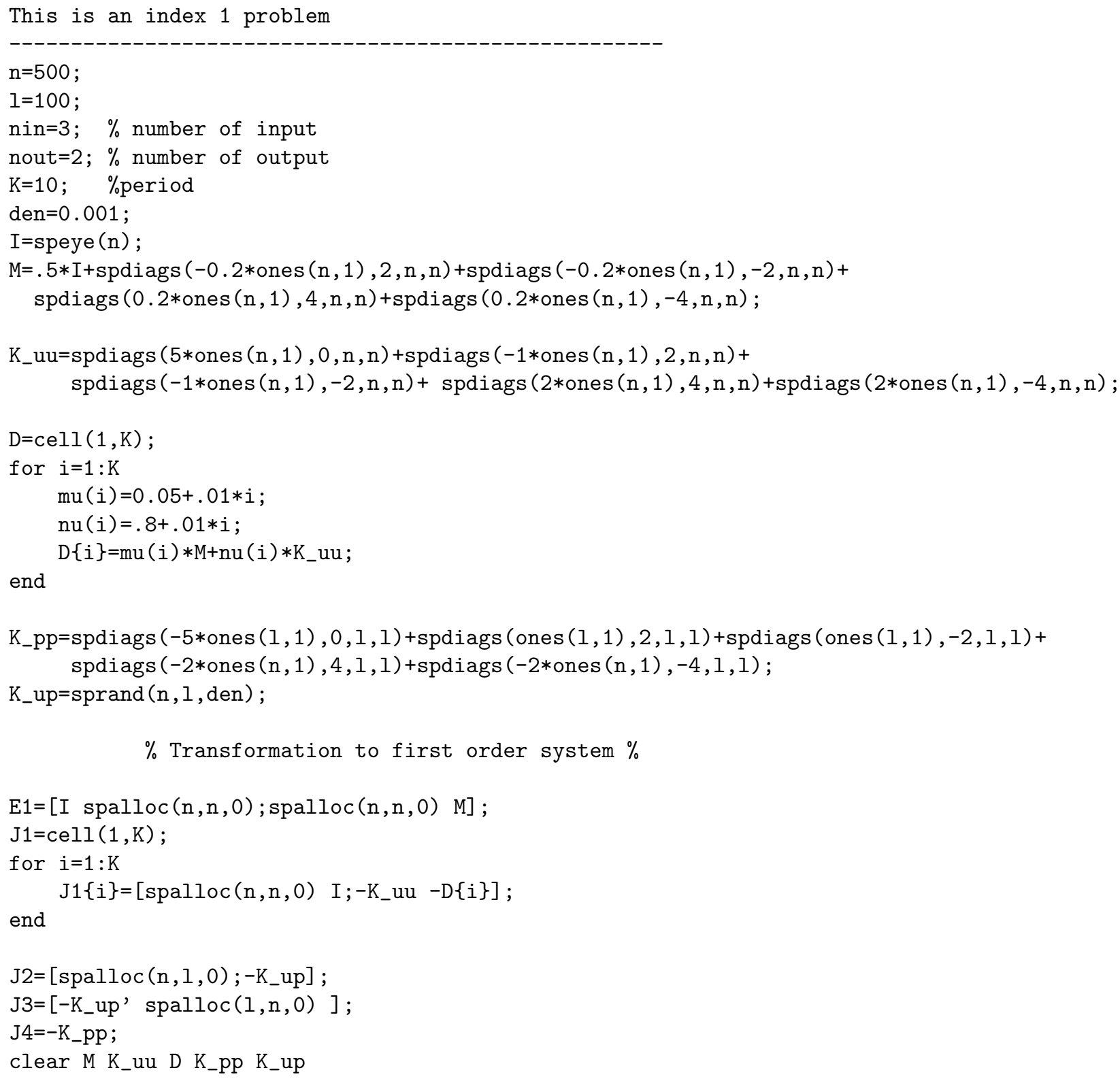




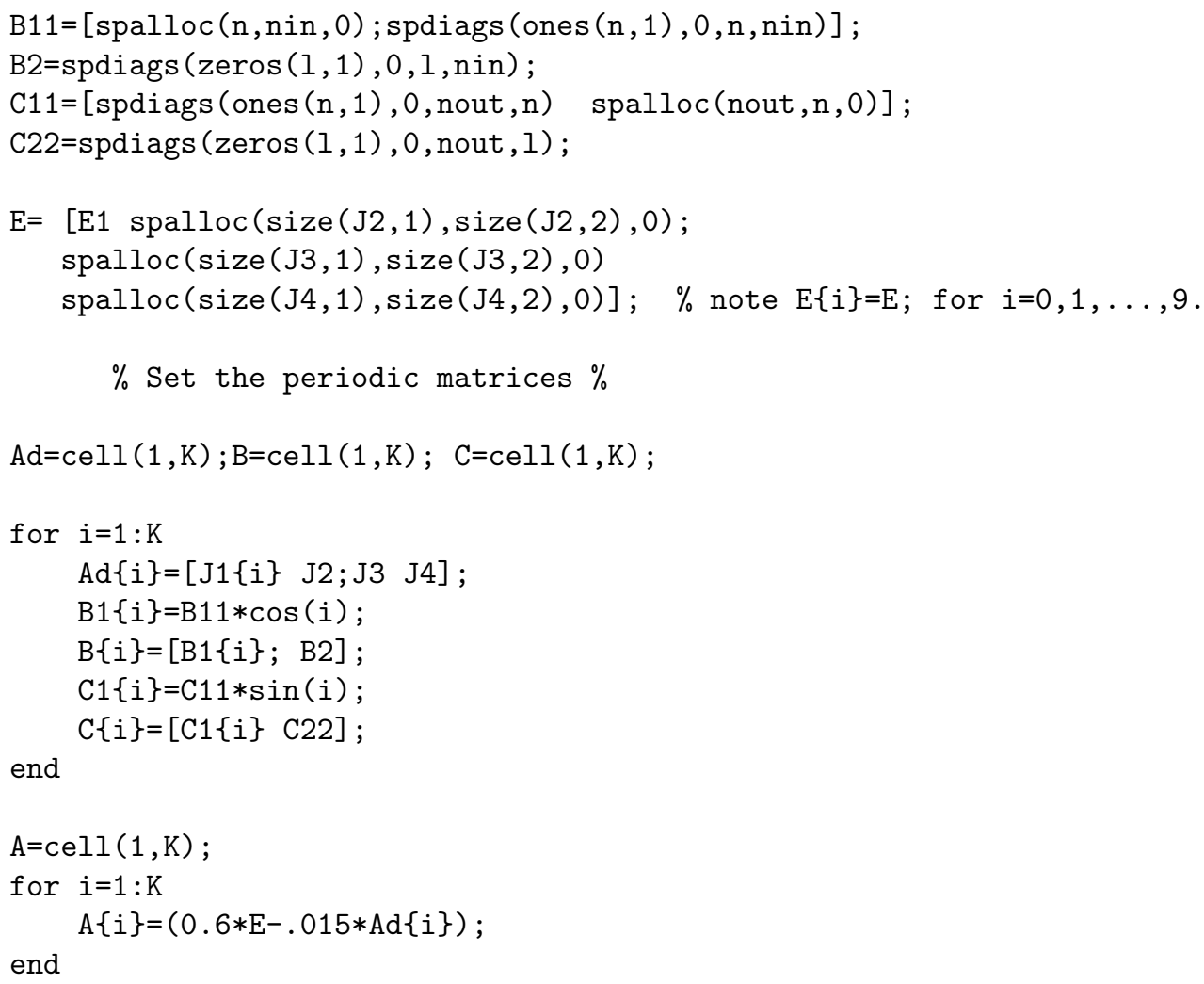




\section{References}

1. P. Benner, M.-S. Hossain, and T. Stykel. Model reduction of periodic descriptor systems using balanced truncation. In P. Benner, M. Hinze, and J. ter Maten, editors, Model Reduction in Circuit Simulation, volume 74 of Lecture Notes in Electrical Engineering, pages 187-200, Springer-Verlag, Berlin, Heidelberg, 2011.

2. P. Benner, M.-S. Hossain, and T. Stykel. Low-rank iterative methods for periodic projected lyapunov equations and their application in model reduction of periodic descriptor systems. Numerical Algorithms, 67(3):669-690, 2014.

3. P. Benner and I.V. Sokolov. Partial realization of descriptor systems. Systems Control Lett., 55(11):929-938, 2006.

4. S. Bittanti and P. Colaneri. Invariant representations of discrete-time periodic systems. Automatica, 36:1777-1793, 2000.

5. S. L. Campbell and C. D. Meyer. Generalized Inverses of Linear Transformations. SIAM, Philadelphia, PA, 2009.

6. E.K.-W. Chu, H.-Y. Fan, and W.-W. Lin. Projected generalized discrete-time periodic Lyapunov equations and balanced realization of periodic descriptor systems. SIAM J. Matrix Anal. Appl., 29(3):982-1006, 2007.

7. M. Farhood, C. Beck, and G.E. Dullerud. Model reduction of periodic systems: a lifting approach. Automatica, 41:1085-1090, 2005.

8. G.H. Golub and C.F. Van Loan. Matrix Computations. 3rd ed. The Johns Hopkins University Press, Baltimore, London, 1996.

9. M.-S. Hossain. Numerical Methods for Model Reduction of Time-Varying Descriptor Systems. Ph.D. thesis, TU Chemnitz, Germany, 2011. Available from http: //nbn-resolving.de/urn:nbn:de:bsz:ch1-qucosa-74776.

10. M. Kono. Invertibility of linear time-varying discrete-time systems. Systems Control Lett., 1(2):148-153, 1981.

11. D. Kressner. Large periodic Lyapunov equations: algorithms and applications. In Proc. of ECC03, Cambridge, UK, pages 951-956, 2003.

12. D. Kressner. An efficient and reliable implementation of the periodic QZ algorithm. In IFAC Workshop on Periodic Control systems, Cernobbio-Como, Italy, August 27-28, 2001.

13. Y.-C. Kuo, W.-W. Lin, and S.-F. Xu. Regularization of linear discrete-time periodic descriptor systems by derivative and proportional state feedback. SIAM J. Matrix Anal. Appl., 25(4):1046-1073, 2004.

14. F.L. Lewis. Fundamental, reachability, and observability matrices for discrete descriptor systems. IEEE Trans. Automat. Control, AC-30:502-505, 1985.

15. V. Mehrmann. A step toward a unified treatment of continuous and discrete-time control problems. Linear Algebra Appl., 241-243:749-779, 1996.

16. M. Nakhla and E. Gad. Efficient model reduction of linear periodically time-varying systems via compressed transient system function. IEEE Trans. Automat. Control, 52(6):1188-1204, 2005

17. B. Park and E.I. Verriest. Canonical forms of discrete linear periodically time-varying systems and a control application. In Proc. of the 28th Conference on Decision and Control, Tampa, FL, pages 1220-1225, Dec 1989.

18. T. Penzl. A cyclic low rank Smith method for large sparse Lyapunov equations. SIAM J. Sci. Comput., 21:1401-1418, 2000.

19. A. M. Perdon, G. Conte, and S. Longhi. Invertibility and inversion of linear periodic systems. Automatica, 28:645-648, 1992.

20. R. Sincovec, A. Erisman, E. Yip, and M. Epton. Analysis of descriptor systems using numerical algorithms. IEEE Trans. Automat. Control, 26(1):139-147, 1981.

21. R.A. Smith. Matrix equation $X A+B X=C$. SIAM J. Appl. Math., 16:198-201, 1968.

22. J. Sreedhar and P. Van Dooren. Periodic descriptor systems: solvability and conditionability. IEEE Trans. Automat. Control, 44(2):310-313, 1999.

23. J. Sreedhar and P. Van Dooren. Periodic Schur form and some matrix equations. In U. Helmke, R. Mennicken, and J. Saurer, editors, Systems and Networks: Mathematical Theory and Applications, Proc. Int. Symposium MTNS '93, Regensburg, Germany, August 2-6, 1993, pages 339-362. Akademie Verlag, Berlin, FRG, 1994. 
24. T. Stykel. Low-rank iterative methods for projected generalized Lyapunov equations. Electron. Trans. Numer. Anal., 30:187-202, 2008.

25. T. Stykel and V. Simoncini. Krylov subspace methods for projected Lyapunov equations. Appl. Numer. Math., 62:35-50, 2012.

26. M.M Uddin. Model Reduction for Piezo-Mechanical Systems using Balanced Truncation. Master thesis, Department of Mathematics, Stockholm University, Stockholm, Sweden, 2011. Available from http://nbn-resolving.de/urn:nbn:de:bsz: ch1-qucosa-78227.

27. P. Van Dooren. The computation of the Kronecker's canonical form of a singular pencil. Linear Algebra Appl., 27:103-140, 1979.

28. A. Varga. Computation of Kronecker-like forms of a system pencil: Applications, algorithms and software. Technical Report R181-95, DLR-Oberpfaffenhofen, Institute for Robotics and System Dynamics, P.O.B. 1116, 82230 Wessling, Germany, January 1995.

29. A. Varga. Balancing related methods for minimal realization of periodic systems. Systems Control Lett., 36:339-349, 1999.

30. A. Varga. Balancing truncation model reduction of periodic systems. In Proc. CDC'2000, Sydney, Australia, volume 3, pages 2379-2384, 2000.

31. A. Varga. Computation of generalized inverses of periodic systems. In Proc. of the 2004 IEEE Conference on Decision and Control(CDC), Paradise Island, Bahamas, volume 5, pages $5397-5402,2004$.

32. A. Varga. Computation of Kronecker-like forms of periodic matrix pairs. In Proc. of Mathematical Theory of Networks and Systems (MTNS 2004, Leuven, Belgium, July 5-9, 2004), 2004

33. A. Varga. A Periodic Systems Toolbox for MATLAB. In Proc. of IFAC'05 World Congress, Prague, Czech Republik, July 3-8, pages 474-474, 2005.

34. A. Varga. An overview of recent developments in computational methods for periodic systems. In Proc. of the Third IFAC Workshop on Periodic Control Systems, St. Petersburg, Russia, August 29-31, 2007.

35. A. Varga and P. Van Dooren. Computing the zeros of periodic descriptor systems. Systems Control Lett., 50:371-381, 2003. 